\title{
Diseño Exploratorio Secuencial (DEXPLOS), procedimiento e instrumento para el análisis de narrativas históricas escolares desde la perspectiva de género
}

Datos de contacto:

Delfín Ortega-Sánchez Universidad de Burgos dosanchez@ubu.es

Davinia Heras-Sevilla Universidad de Burgos dheras@ubu.es

Recibido: 31/01/2021 Aceptado: 29/03/2021

\author{
Delfín ORTEGA-SÁNCHEZ \\ Davinia HERAS-SEVILLA
}

\section{RESUMEN}

Este estudio ofrece una descripción detallada del diseño y procedimiento metodológico aplicado en el análisis de las representaciones sociales sobre la Historia en las narrativas del alumnado de Educación Primaria. La investigación se posiciona en los enfoques mixtos de investigación, en particular, en los diseños exploratorios secuenciales (DEXPLOS) derivativos, en los que el proceso de investigación combina la recolección, codificación uniforme, categorización y análisis descriptivo e interpretativo de datos cualitativos (fase 1) con datos cuantitativos, obtenidos sobre la base de los primeros, a través de la construcción ad hoc de una escala categorial de medición de las unidades de análisis (fase 2). Los resultados obtenidos evidencian la validez y operatividad del diseño metodológico e instrumento propuesto para el análisis y reflexión sobre los enfoques curriculares dominantes en la enseñanza y aprendizaje de la Historia escolar en esta etapa educativa. De acuerdo con los resultados obtenidos en la primera aplicación de esta metodología e instrumento, la transmisión de discursos históricos estereotipados consolida la reproducción de narrativas y la selección predeterminada de ciertos actores protagónicos por parte del alumnado. Esta selección determina, igualmente, la conformación de identidades sociales e históricas que, en caso de proponer a las mujeres en estadios subsidiarios y dependientes, impulsan la exclusión de aquellos que "no hacen historia".

PALABRAS CLAVE: Representaciones sociales; historia escolar; narrativas históricas; género; Educación Primaria; DEXPLOS 


\title{
Sequential Exploratory Design (DEXPLOS), procedure and instrument for the analysis of school historical narratives from a gender perspective
}

\begin{abstract}
This study offers a detailed description of the design and methodological procedure applied in the analysis of social representations on History in the narratives of elementary school students. The research is positioned in mixed research approaches, in particular, in derivative exploratory sequential designs (DEXPLOS), in which the research process combines the collection, uniform codification, categorization, and descriptive and interpretative analysis of qualitative data (phase 1) with quantitative data, obtained on the basis of the first, through the ad hoc construction of a categorical scale of measurement of the units of analysis (phase 2). The results obtained show the validity and operability of the methodological design and instrument proposed for the analysis and reflection on the dominant curricular approaches in the teaching and learning of school history at this educational stage. According to the results obtained in the first application of this methodology and instrument, the transmission of stereotyped historical discourses consolidates the reproduction of narratives and the predetermined selection of certain protagonists by students. This selection also determines the shaping of social and historical identities which, in the case of proposing women in subsidiary and dependent stages, encourages the exclusion of those who "do not make history".
\end{abstract}

KEYWORDS: Social Representations; school history; historical narratives; gender; Primary Education; DEXPLOS

\section{Introducción}

La expresión narrativa constituye una de las bases constructivas del conocimiento histórico (McEwan \& Egan, 1995), de la representación, interpretación y organización de la realidad social e histórica, y de la comprensión de la temporalidad e historicidad humanas. Necesita fuentes para su configuración, se incardina en la formulación de finalidades específicas, explica problemas, y se halla condicionada por tiempos y espacios determinados. Por lo tanto, constituye un principio de inteligibilidad y una propuesta de conocimiento (Pesavento, 2000).

Las implicaciones educativas de la narrativa y, en particular, de la histórica, precisan centrar la atención en las claves constructivas del relato, así como en sus propósitos y usos sociales, políticos y educativos. El acto narrativo, soporte organizativo e interpretativo para la representación de la realidad social e histórica, demanda el empleo de habilidades cognitivas de orden superior como el análisis -clasificación e interpretación de contenidos-y la evaluación -selección, justificación y reflexión-, y el desarrollo de habilidades de interpretación e imaginación creativa a través de la experiencia y de juicios morales del presente (Ortega-Sánchez \& Pagès, 2018). La narrativa histórica representa, en consecuencia, una de las formas más 
evidentes de exteriorizar la conciencia histórica, esto es, la direccionalidad del sentido otorgado a las relaciones históricas y temporales pasado/presente/futuro (Cartes et al., 2019). La competencia narrativa habría de entenderse, en este sentido, como la capacidad de comprender el pasado y de materializar la historicidad del presente para su proyección hacia futuros posibles cada vez más inclusivos y globales. Esta competencia asume, por tanto, la comprensión de la forma en que los y las historiadoras explican el pasado, a partir de los mecanismos constructivos del relato histórico (Seixas \& Morton, 2013).

Para analizar y reflexionar sobre las narrativas históricas del alumnado partimos de la teoría crítica (McLaren \& Kincheloe, 2015; Apple, 2014) como eje teóricoreflexivo en torno a las relaciones desiguales de género. Esta perspectiva teórica contribuye a comprender el grado en que la escuela, en tanto agente socializador tradicional, se aproxima o se aleja de la racionalidad constructiva sociocultural del concepto de género. La escuela, en efecto, constituye un espacio hegemónico donde el poder y las ideologías se disputan el dominio de las estructuras. En este sentido, la enseñanza de la historia escolar tradicionalmente se ha centrado en la importancia de los hombres que han ostentado y ejercido el poder (Azorín, 2015). Las narrativas y las voces ajenas a los ámbitos protagónicos de acción social no están presentes ni son visibles, por estar fuera de las estructuras patriarcales hegemónicas y de las élites dominantes (Balteiro \& Roig-Marín, 2015).

De acuerdo con Sáiz y López (2016), se identifica la existencia de una "mediación de la narrativa tradicional en los que los futuros docentes consideran que debe aprender su alumnado" (p. 138). Desde esta perspectiva, el profesorado podría resultar determinante en la transmisión y perpetuación de los estereotipos, los prejuicios y las estructuras sociales, políticas y culturales de marginación por razón de género. Esto se debe a que los y las docentes tienden a enseñar bajo el mismo enfoque curricular en el que ellos y ellas mismas se formaron (Crocco, 2018; Vavrus, 2009; Stanley, 2010) y, en cuyos espacios tradicionalmente patriarcales y hegemónicos, las mujeres y su historia estaban ausentes (Fernández Valencia, 2015). En efecto, los estudios coinciden en afirmar que, cuando se incluye a las mujeres en la enseñanza, es desde una posición anecdótica o aditiva a un discurso narrativo dominado por hombres (Asián et al., 2015; Pagès \& Sant, 2012).

Considerando la competencia narrativa como uno de los componentes esenciales del desarrollo del pensamiento histórico y social en la enseñanza y aprendizaje de la Historia (Sant et al., 2014), este estudio ofrece, de una parte, un diseño metodológico y un modelo procedimental para analizar, desde la perspectiva de género, las representaciones históricas en las narrativas escolares. De otra, busca describir con exhaustividad su validez, a partir de los resultados obtenidos en la investigación de Ortega-Sánchez et al. (2020). Con estos objetivos, se formula la siguiente pregunta de investigación: ¿De qué forma y en qué grado puede medirse la contribución de las narrativas históricas de los y las estudiantes a la consolidación de identidades de género hegemónicas o, en cambio, a la construcción de identidades de género diversas y plurales? 


\section{Diseño}

El diseño metodológico e instrumental propuesto se posiciona en los enfoques mixtos de investigación, en particular, en los diseños exploratorios secuenciales (DEXPLOS) derivativos (Creswell, 2009), en los que el proceso de investigación combina la recolección, codificación uniforme, categorización y análisis descriptivo e interpretativo de datos cualitativos - contenido manifiesto- (fase 1), con datos cuantitativos, obtenidos sobre la base de los primeros, a través de la construcción ad hoc de una escala categorial de medición de las unidades de análisis —narrativas históricas-(fase 2).

Este enfoque parte de la clásica definición del análisis de contenido, a cuyos principios de objetividad, sistematicidad y cuantificación de contenidos manifiestos se añade la inferencia de los datos obtenidos al contexto de referencia. La inferencia constituye, precisamente, el propósito nuclear del análisis de contenido cuantitativo (ACC), esto es, la realización, a partir de los datos, de "inferencias válidas y replicables, que puedan aplicarse a su contexto" (Cea, 1996, p. 352), una vez cumplidas las condiciones de validez y de fiabilidad exigidas en el proceso de medición. En este sentido, Cea D’Ancona afirma que

En el análisis de contenido cuantitativo, el énfasis no recae en los aspectos semánticos o sintácticos de los textos, sino en la cuantificación de sus integrantes (palabras, expresiones, frases, temas); es decir, en la medición de su frecuencia de aparición en el texto. Esta cuantificación permite, precisamente, la comparación de los contenidos de diferentes documentos y, sobre todo, la inferencia (p. 352).

El presente diseño metodológico e instrumental se adscribe, en efecto, a la técnica del ACC, por cuanto no se limita a la descripción textual (características textuales), sino que su interpretación (significación de las características textuales) se sostiene, por tanto, en la inferencia de los resultados.

A diferencia del procedimiento analítico del contenido temático o cualitativo, basado en la identificación, sistematización y análisis de tópicos/sub-tópicos categoriales observados, el análisis textual evaluativo, en la línea de la perspectiva metodológica de Mayring (2010), implica la evaluación, clasificación y valoración del contenido mediante categorías evaluativas cuali-cuantitativas. Desde esta perspectiva, en el análisis cualitativo evaluativo o cuantitativo del contenido aplicado, "los investigadores o codificadores evalúan los datos y construyen categorías [evaluativas], cuyas características suelen anotarse como números ordinales o niveles" (Kuckartz, 2014, p. 88). De esta forma, este análisis escalado transforma el texto en números y, en última instancia, conduce a un análisis estadístico (Kuckartz, 2014, p. 88). 


\section{Procedimiento}

La generación y análisis de cada variable de estudio y categoría evaluativa siguió las fases procesuales que pueden observarse en la figura 1.

\section{Figura 1}

Proceso de análisis cualitativo evaluativo de textos en siete fases.

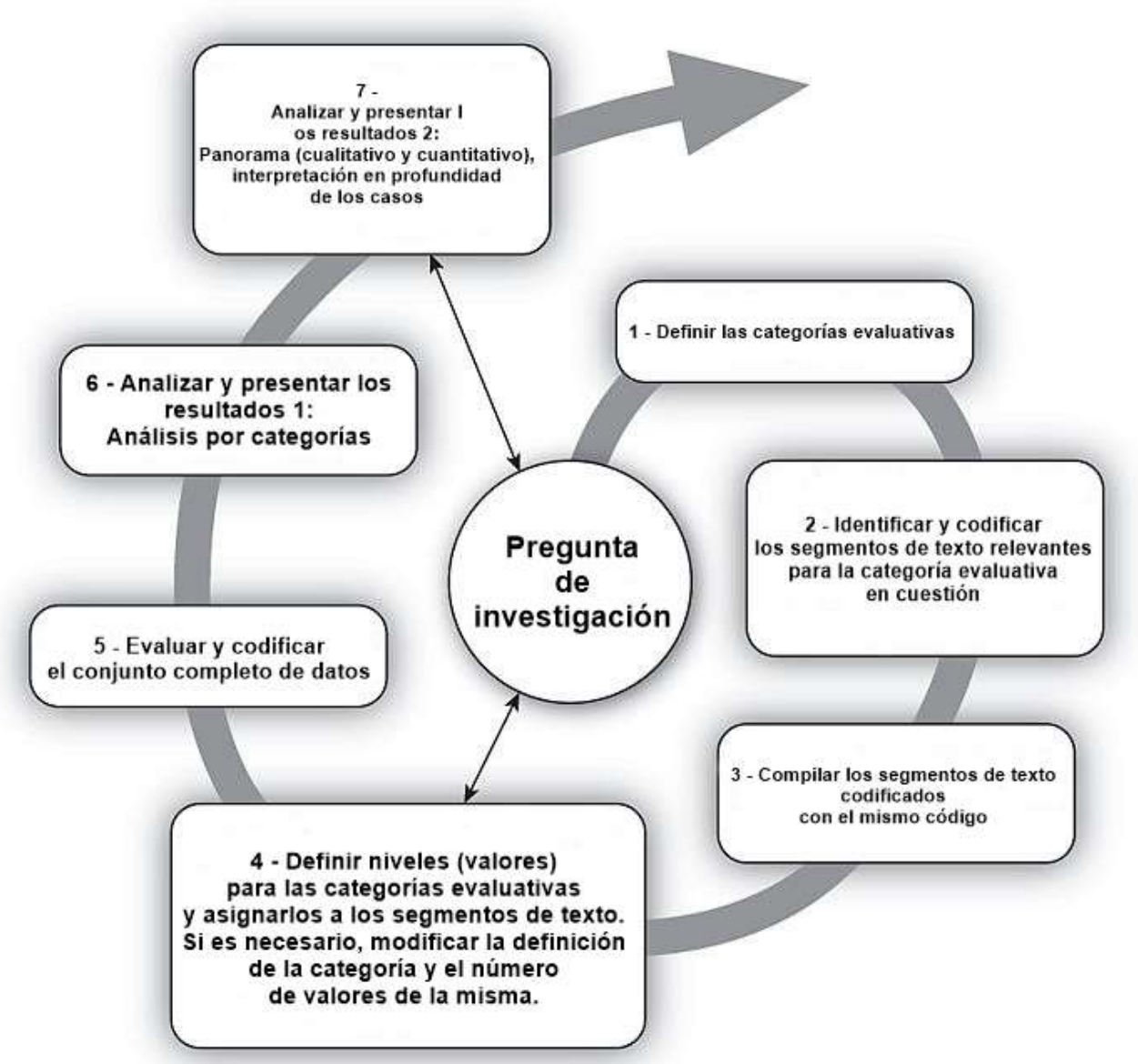

Fuente: Traducción propia a partir de Kuckartz (2014, p. 89).

Al proceso analítico de Kuckartz (2014) se añadieron, de forma adaptada e integrada, las fases definidas por D’Ancona (1996) (Figura 2). 


\section{Figura 2}

Fases principales en la realización de un análisis de contenido cuantitativo.

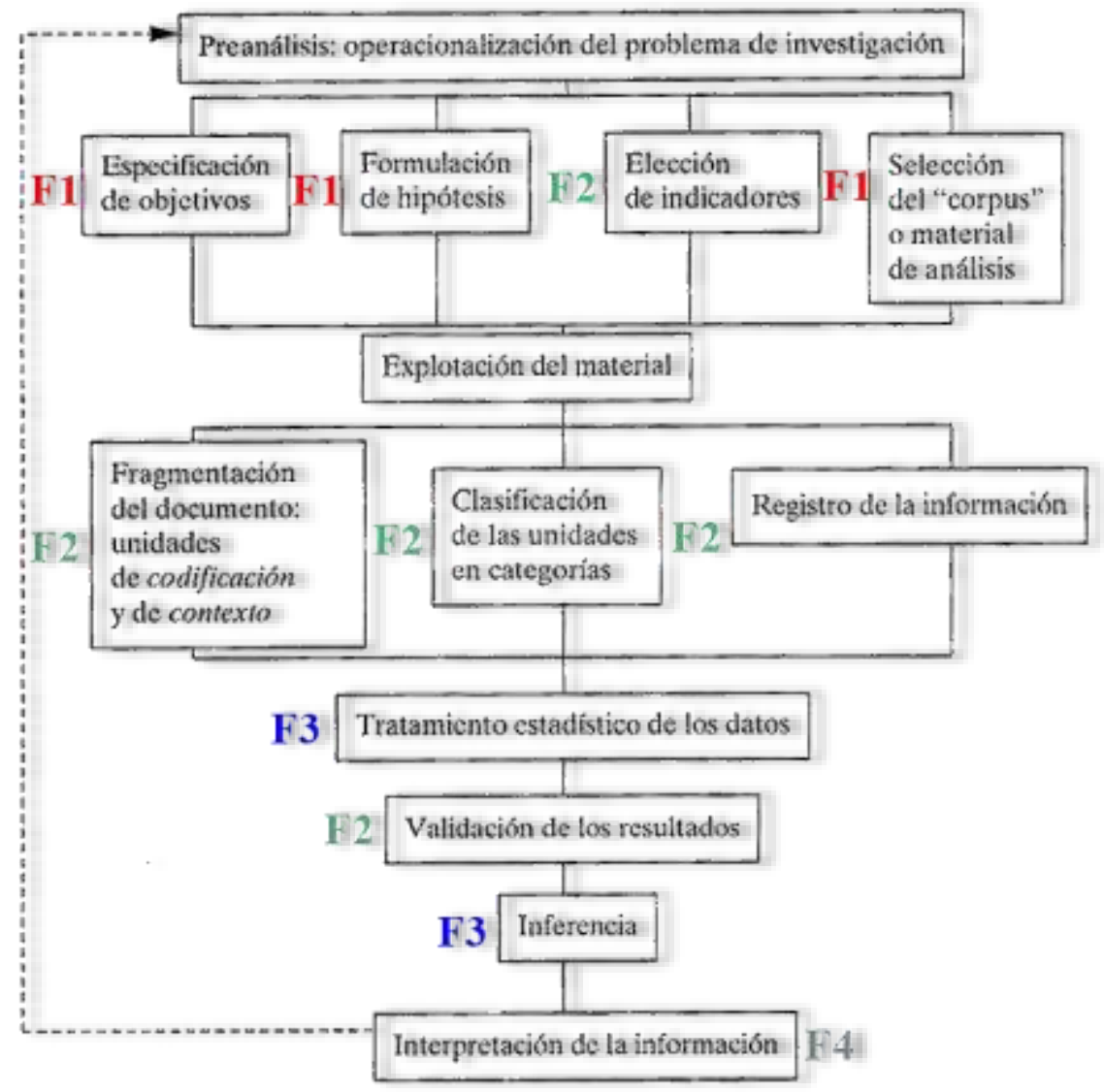

Fuente: Adaptación de D’Ancona (1996, p. 354).

De acuerdo con las fases de diseño metodológico y analítico de Kuckartz (2014) y D'Ancona (1996), se definieron cuatro fases procesuales $\left(\mathrm{F}_{1}-\mathrm{F}_{4}\right)$, integradas en dos fases operacionalizadoras generales $\left(\mathrm{FO}_{1} \mathrm{y} \mathrm{FO}_{2}\right)$.

En primer lugar $\left(\mathrm{F}_{1}\right)$, se procedió a operacionalizar el problema de investigación mediante la formulación de sus objetivos e hipótesis, y la selección documental (muestra poblacional narrativa), a partir de la aplicación de un muestreo intencional o de conveniencia, en función de las posibilidades de acceso del equipo investigador al campo de estudio, las características de la población documental, su grado de adecuación a los objetivos de investigación y el tipo de inferencia que se buscaba realizar.

En segundo lugar $\left(\mathrm{F}_{2}\right)$, se definieron las unidades de codificación o de registro a partir de palabras clave, temas de interés para el problema de investigación y personajes o actores narrativos. Después de realizar una lectura detenida de las unidades documentales, se procedió a codificar, de forma inductiva, los textos 
narrativos. De acuerdo con los principios metodológicos fenomenológicos y el modelo de análisis proposicional de Campos (2005), cada proposición textual fue dividida en segmentos, con el objetivo de extraer los principales componentes conceptuales y excluir las relaciones lógicas preposicionales y verbales, los artículos determinados, indeterminados y demostrativos, y las conjunciones y conectores textuales. Finalizada la codificación inductiva, se generaron las variables explicativas (indicadores) y las categorías centrales a partir de las intersecciones relacionales identificadas entre códigos y su agrupamiento conceptual en clústeres naturales. En este proceso, también fueron delimitadas las unidades de contexto, con el fin de alcanzar la interpretación semántica de las unidades de codificación en su lugar de aparición. Seguidamente, las unidades de registro fueron finalmente asignadas a una de las variables y categorías emergentes generadas por el sistema clasificatorio de codificación.

En la definición teórico-inductiva de las variables y categorías se aseguró el cumplimiento de los criterios de medición (exhaustividad, exclusividad, precisión, pertinencia o adecuación categorial a los objetivos de la investigación, y homogeneidad o capacidad de respuesta categorial a un único principio de clasificación), y su viabilidad tras la aplicación piloto en una muestra procedente de la misma población documental $(n=15)$.

En esta misma fase, el contenido de las unidades de registro fue validado mediante la confirmación de su posible asignación a una única variable y categoría de análisis, la existencia de una clara capacidad explicativa de las categorías por cada una de las variables de estudio que las conformaban (validez de contenido), y el grado de similitud semántica entre las unidades de codificación y su clasificación categórica (validez semántica). Igualmente, las categorías empíricas del estudio fueron confirmadas de acuerdo con las hipótesis de investigación. A la comprobación de la adecuación del sistema categorial propuesto (validez) se añadió la comprobación de la consistencia del procedimiento de medición (fiabilidad). La fiabilidad procedimental se garantizó con la obtención de un alto grado de acuerdo entre codificadores (equipo de investigación -codificador 1- e investigador externo -codificador 2-) en relación con los criterios de estabilidad (ausencia de ambigüedad en la asignación de las unidades de registro a una variable y categoría) y reproductibilidad (acuerdo inter-investigadores en la codificación narrativa) (Krippendorff, 1990). La fiabilidad de codificación y categorización (Muñoz \& Montoro, 2005) fue confirmada, además, mediante la aplicación del índice $\pi$ de Scott $(\pi=0.81)$.

En tercer lugar $\left(\mathrm{F}_{3}\right)$, de acuerdo con los indicadores (variables) contemplados, se registraron y cuantificaron los datos cualitativos obtenidos para proceder a su tratamiento estadístico, cuyo interés fue "más allá de la mera descripción frecuencial de las unidades de codificación por separado, [lo que exigió] la previa codificación numérica del contenido manifiesto del material verbal" (D'Ancona, 1996, p. 365). El análisis de los datos categoriales o temáticos obtenidos comenzó con el análisis univariante de sus variables de estudio y finalizó con la aplicación de pruebas inferenciales bivariadas.

Finalmente, en cuarto lugar $\left(\mathrm{F}_{4}\right)$, se interpretaron los resultados obtenidos, 
considerando su aplicabilidad mediante la identificación de los patrones culturales, las intenciones y los efectos de los mensajes narrativos en la construcción de las identidades de género, evaluada en términos de presencia/ausencia de actores sociales.

En la $\mathrm{F}_{1}$, la obtención de las narrativas adaptó el método diseñado por Létourneau y Caritey (2008) de la hoja en blanco (Sant et al., 2014a), para cuya aplicación se empleó un cuestionario con una única pregunta de respuesta abierta (¿Qué sabes de la Historia de España?). Partiendo de la valoración de este tipo de preguntas como versiones válidas de la técnica de la entrevista (Esses \& Maio, 2002), el procedimiento para determinar la validez de contenido del instrumento consistió en la valoración, en un rango de 1 a 5 puntos, del potencial informativo, de la eficacia esperada y de la operatividad de la cuestión por 3 especialistas en Didáctica de la Historia, y en la revisión de la literatura científica en la que se aplica el mismo instrumento (González-Monfort et al., 2015; Sant et al., 2014; Létourneau \& Caritey, 2008). Los resultados mostraron la existencia de un alto índice de concordancia interjueces y una significativa homogeneidad entre los rangos asignados $(W=0.791, p=$ 0.000), lo que confirmó la validez de su contenido.

La recogida de datos se desarrolló en el entorno natural de los y las participantes (cuatro aulas de sexto curso de dos centros educativos de Educación Primaria) entre mayo de 2018 y junio de 2019. Los y las estudiantes dispusieron de un máximo de dos páginas para redactar sus respuestas. Con el objeto de ceder la mayor libertad posible, no se les demandó el seguimiento de ninguna instrucción en la construcción de sus textos.

En la $\mathrm{FO}_{2}$, generadora de la escala categorial de medición, el estudio ajusta su adscripción a los principios metodológicos de los diseños no experimentales transversales, descriptivos y correlacionales ex post facto, en los que las variables independientes no son manipuladas, o sobre las que no se influye o interviene porque ya han sucedido (Hernández et al., 2010).

\section{Instrumento}

Mediante el análisis del contenido narrativo manifiesto (ACM), realizado a través de la comparación constante de los datos (Libarkin \& Kurdziel, 2002), de su análisis temático, palabras clave y personajes o actores narrativos, y de la identificación de patrones de respuesta textual (Denzin \& Lincoln, 2018), cada unidad de registro y contexto/significado seleccionada fue asignada a una de las diez variables y tres categorías emergentes (Tabla 1). 


\section{Tabla 1}

Escala categorial, variables y niveles de progresión

\begin{tabular}{|c|c|c|c|}
\hline$C$ & $V$ & $N P r$. & Descriptor \\
\hline \multirow[b]{2}{*}{1} & $\begin{array}{c}\text { Político- } \\
\text { institucional }\end{array}$ & 1 & $\begin{array}{l}\text { La narrativa histórica se articula a partir de hechos, sucesos y } \\
\text { eventos políticos y/o militares, presentados como } \\
\text { constructores de la historia de España. }\end{array}$ \\
\hline & Socio-cultural & 2 & $\begin{array}{l}\text { Desde una perspectiva inclusiva, se describen las estructuras } \\
\text { de lo social y las expresiones culturales de la vida cotidiana } \\
\text { de hombres y mujeres, con independencia de su adscripción } \\
\text { social (elites / grupos sociales desfavorecidos). }\end{array}$ \\
\hline \multirow{4}{*}{2} & $\begin{array}{l}\text { Cuasi- } \\
\text { personaje }\end{array}$ & 1 & $\begin{array}{l}\text { La narrativa aparece protagonizada por países y/o entidades } \\
\text { político-institucionales humanizadas en proto-estructuras de } \\
\text { aparente dominio masculino. }\end{array}$ \\
\hline & $\begin{array}{l}\text { Individual- } \\
\text { colectivo }{ }^{\lambda}\end{array}$ & 2 & $\begin{array}{l}\text { La narrativa presenta una tendencia a priorizar las figuras de } \\
\text { protagonistas históricos masculinos o articularse a partir de } \\
\text { grupos de individuos masculinos, constructores del relato } \\
\text { histórico-social. Las mujeres, como colectivo y actor social, y } \\
\text { otros grupos sociales son invisibles. }\end{array}$ \\
\hline & $\begin{array}{l}\text { Individual- } \\
\text { colectivo }+\end{array}$ & 3 & $\begin{array}{l}\text { La narrativa visibiliza las figuras protagónicas históricas } \\
\text { femeninas o se articula a partir de grupos de individuos } \\
\text { femeninos de forma añadida o aditiva al eje narrativo } \\
\text { principal. }\end{array}$ \\
\hline & Mixto $\delta^{\lambda}+$ & 4 & $\begin{array}{l}\text { La narrativa se articula a partir de la acción protagónica de } \\
\text { hombres y mujeres, mediante la concreción de sus } \\
\text { aportaciones y experiencias históricas al fenómeno social } \\
\text { descrito. }\end{array}$ \\
\hline \multirow{4}{*}{3} & $\begin{array}{l}\text { Político- } \\
\text { militar }\end{array}$ & 1 & $\begin{array}{l}\text { La acción social de los y las protagonistas históricas } \\
\text { seleccionadas se desarrolla en escenarios políticos, } \\
\text { diplomáticos y/o institucionales, o en escenarios de } \\
\text { naturaleza militar, basados en el enfrentamiento armado a } \\
\text { escala local y/o internacional. }\end{array}$ \\
\hline & Económico & 2 & $\begin{array}{l}\text { La acción social de los y las protagonistas históricas } \\
\text { seleccionadas se desarrolla en escenarios de ámbito local o } \\
\text { global en torno a intereses y/o a la toma de decisiones de } \\
\text { índole económica. }\end{array}$ \\
\hline & Cultural & 3 & $\begin{array}{l}\text { La acción social de los y las protagonistas históricas } \\
\text { seleccionadas se desarrolla en el ámbito de la cultura } \\
\text { artístico-patrimonial. }\end{array}$ \\
\hline & $\begin{array}{l}\text { Privado- } \\
\text { familiar }\end{array}$ & 4 & $\begin{array}{l}\text { La acción social de los y las protagonistas históricas } \\
\text { seleccionadas se desarrolla en espacios privados y/o } \\
\text { familiares. }\end{array}$ \\
\hline
\end{tabular}

C1: Categoría 1 (enfoque historiográfico); C2: Categoría 2 (protagonistas históricos); C3: Categoría 3 (ámbitos protagónicos de acción social). V: Variable. NPr.: Nivel de progresión ordinal.

El diseño completo del instrumento, construido ad hoc tras el ACM, consta de 10 variables aleatorias que concretan, de forma integral, los atributos que definen el 
modelo. Para el análisis de las variables 1, 2, 7, 8, 9 y 10, se aplica una escala de 3 puntos de medida ordinal, con un punto máximo, un punto mínimo y un punto medio, acerca de la proximidad narrativa de cada extracto textual a los atributos de la variable de estudio, donde el valor 1 corresponde a un bajo nivel de aproximación narrativa y el valor 3 a un alto nivel de aproximación. En el análisis de las variables 3 , 4, 5 y 6, se aplica, por su parte, una escala ordinal comprendida entre 1 y 3 puntos relativa a las frecuencias y/o recurrencias de aparición numérica de las características de cada variable, donde el valor 1 implica el registro numérico de 0 a 3 protagonistas históricos, el valor 2 la presencia de entre 4 a 6 protagonistas, y el valor de 3 la inclusión de más de 7 personajes con las características correspondientes a la variable de estudio.

Por último, cada variable categórica se presenta asociada a un nivel jerárquico de progresión ordinal o hipótesis progresiva con 4 niveles de complejidad, donde 1 equivale a la adopción de enfoques hegemónicos y androcéntricos de la historia enseñada, y 4 a enfoques de naturaleza socio-crítica e inclusiva, dirigidos a la educación para una ciudadanía democrática, diversa y plural. Este modelo empírico, próximo a los principios de la teoría fundamentada y de la fenomenología de orientación inductiva, fue diseñado a partir de los trabajos de González-Monfort et al. (2015), Ortega-Sánchez (2017, 2019) y Sant et al. (2014a, 2014b).

La confiabilidad, credibilidad y transferibilidad de la escala categorial resultante fue garantizada por el equipo investigador a través de la discusión, reflexión, redefinición y posterior ajuste de las categorías a la información recabada con anterioridad a su tratamiento estadístico. Para determinar su precisión, estabilidad y consistencia interna, comprobar la correlación entre variables y su capacidad para medir un mismo constructo, y evitar errores aleatorios en la medición específica de sus atributos, se calculó el coeficiente alfa de Cronbach. Atendiendo a la oposición entre los atributos existentes entre las variables de estudio, se procedió a su recodificación y ajuste con anterioridad al cálculo. El análisis devolvió un nivel adecuado $(\alpha=.83)$ para el conjunto de las variables categóricas.

Asimismo, con el fin de obtener una óptima saturación y concreción informativa en cada categoría, y de profundizar en las representaciones del conocimiento histórico del alumnado, se aplicó un conteo de palabras clave estructurantes mediante el registro de la frecuencia de aparición y, en consecuencia, grado de importancia otorgado a los nombres propios y/o colectivos de protagonistas históricos y de países incluidos, explícitamente, en las narrativas históricas. Con el objetivo de obtener núcleos centrales explicativos de representación (Moliner \& Abric, 2014), este indicador ha sido empleado en recientes trabajos sobre representaciones sociales del alumnado de Educación Primaria (Conde \& Armas, 2019).

\section{Análisis de datos}

El corpus documental $(n=96)$ fue identificado mediante códigos alfanuméricos $\left(\mathrm{C}_{\mathrm{n}^{\mathrm{o}}}\right.$ de centro_ $\mathrm{N}_{\mathrm{n}^{\mathrm{o}}}$ de estudiante_ $\mathrm{A}_{\mathrm{n} \text { o }}$ de aula $)$. Se obtuvo un total de 384 extractos narrativos medidos en cada una de las tres categorías y variables establecidas. 
Para la organización, codificación y posterior análisis de los datos cualitativos se ha utilizado el software MAXQDA, y, para el cálculo de frecuencias y porcentajes de palabras clave, el software de datos cualitativos ATLAS.ti v. 7.5.4.

Los análisis cuantitativos descriptivos (medidas de posición central -moda-) y, comprobada la ausencia de parámetros de normalidad de las variables - prueba de Kolmogorov-Smirnov $(n>50, p<.05)-\mathrm{y}$ de homocedasticidad (prueba de Levene), los análisis inferenciales de los datos obtenidos (tablas de contingencia, ANOVA de Kruskal-Wallis, prueba $U$ de Mann-Whitney y correlaciones de Spearman) fueron realizados con el propósito de identificar la existencia de diferencias estadísticamente significativas entre aulas y centros educativos, y una potencial interdependencia entre las variables categóricas de medida ordinal, a partir del análisis de sus covarianzas. Esta combinación de análisis pretende alcanzar una comprensión integral de los resultados obtenidos. Para el tratamiento de los datos cuantitativos se utilizó el software SPSS v.24.

Mediante este modelo metodológico, se asume la posibilidad de realizar inferencias textuales y de identificar diferencias estadísticamente significativas en función de las variables de estudio, de forma similar a las que pudieran extraerse con cualquier otra técnica cuantitativa mediante cuestionario. Desde esta perspectiva, coincidimos con Neuendorf (2002) y Andréu (2000) en afirmar que, a través de lo que las personas "dicen o escriben, se expresan sus intenciones, actitudes, sus conocimientos, su interpretación de la situación..., y cualquier valor [susceptible de codificación] como una variable de un cuestionario" (Andréu, 2000, p. 20). Esta propuesta metodológica busca, de forma integrada, la interpretación textual, el análisis estadístico descriptivo de las variables de estudio sobre el contenido manifiesto, y la realización de inferencias sobre su estado y relación en un momento determinado (Denzin \& Lincoln, 2018).

\section{Discusión y conclusiones}

Los resultados obtenidos en el presente estudio que, por primera vez en el contexto iberoamericano, aplicó este diseño metodológico (Ortega-Sánchez et al., 2020) evidencian su validez y operatividad en el análisis narrativo histórico-social. Estos resultados confirmaron la continuidad y consolidación de enfoques curriculares positivistas, la concepción técnica y lineal en los aprendizajes históricos percibidos, y sus implicaciones en la formación inicial del profesorado de Historia y Ciencias Sociales (Ortega-Sánchez \& Heras-Sevilla, 2020; Ortega-Sánchez \& Pagès, 2018; Moreira et al., 2019). La consistencia y validez de este diseño y procedimiento metodológico ha sido demostrado en análisis narrativos similares desde el ámbito de la literatura infantil (Shahnaz et al., 2020), cuyos resultados son consistentes con los presentados en esta investigación, y en los que se demuestra la ausencia o presencia estereotipada de las mujeres en la construcción de los relatos tanto de naturaleza histórico-social como literaria.

A pesar de la vinculación de las unidades de codificación con sus unidades de contexto naturales, las limitaciones del diseño metodológico, procedimental e instrumental aplicado pasan por la necesidad de triangular los resultados 
cuantitativos obtenidos con otros, de naturaleza puramente cualitativa, capaces de matizar el significado y sentido final de las narrativas históricas escolares. En este sentido, como bien apunta D’Ancona (1996), “aunque la cuantificación ayude a la sistematización y estandarización del análisis textual, no siempre se profundiza en los contenidos no manifiestos de los mensajes" (p. 373).

Parece generalizada la asunción de que el propósito de la enseñanza de la Historia centra su interés en el desarrollo y adquisición de habilidades sociales necesarias para la participación, autónoma y reflexiva, en sociedad. Sin embargo, parece también común la articulación de una enseñanza de la Historia a partir de narrativas acríticas y pseudo-objetivas diseñadas para la definición y justificación de conciencias nacionales (Laville, 1999). Coincidimos con Regina (2019) en afirmar la plena vigencia del texto de Laville sobre la "guerra narrativa" y la intencionalidad contextual de los contenidos históricos escolares.

La transmisión de discursos históricos estereotipados continúa legitimando, en efecto, la reproducción de narrativas y la selección predeterminada de ciertos actores protagónicos por parte del alumnado. Esta selección determina, igualmente, la conformación de identidades sociales e históricas que, en caso de proponer a las mujeres en estadios subsidiarios y dependientes, impulsan la exclusión de aquellos que "no hacen historia" (Sant, 2014a). Considerando la orientación temporal y la competencia narrativa como ejes estructurantes de la Didáctica de la Historia (Cerri, 2013), y valorando la naturaleza constructiva de la categoría sociocultural de género,

Necesariamente habrá que revisar y/o redefinir las finalidades de enseñanza de las ciencias sociales y construir una narrativa no androcéntrica ni heteropatriarcal, que pueda construirse a partir de nuevas formas discursivas con un sentido diferente y que posibilite al profesorado plantearse una reflexión crítica sobre las prácticas sociales y los modelos de ciudadanía (Arangue, 2019, p. 251).

Puede afirmarse, en consecuencia, la necesidad de trabajar las competencias en literacidad crítica en la formación docente y en la enseñanza de las ciencias sociales, con el fin de deconstruir, desde su racionalidad discursiva, no sólo los programas curriculares, sino también los discursos y las prácticas de los y las docentes en torno a su contribución a la pluralización e inclusión social en la historia enseñada. En efecto, el objetivo que debe impulsar la erradicación de las estructuras androcéntricas y hegemónicas habría de pasar por el análisis de las prácticas docentes, en las que la justicia social ha de posicionarse como uno de los valores intrínsecos del proceso de enseñanza y aprendizaje de la historia escolar. En efecto, "la historia enseñada en escuelas e institutos debería dotar de criterios para comprender problemas sociales (...), aprendiendo a (...) cuestionar cualquier identidad esencialista" (Sáiz \& López, 2016, p. 139).

Continúa siendo necesario, en consecuencia, el diseño, implementación y evaluación de programas para la formación del profesorado dirigidos a la visibilización de mujeres en la construcción del conocimiento histórico-social. Un excelente ejemplo se encuentra en el proyecto @Filosoclips, orientado a la identificación e incorporación de las mujeres filósofas en el currículo del Bachillerato español (Triviño-Cabrera et al., 2020). Con este propósito, esta experiencia didáctica, 
en la que se utilizaron vídeos de cantantes de éxito como recurso, buscó

Transformar el currículo oficial de Filosofía en un currículo equitativo para el desarrollo de habilidades críticas, creativas, empáticas y de empoderamiento en los chicos y chicas para que sean conscientes de su responsabilidad social en la creación de una sociedad justa y equitativa (p. 13).

En una línea similar, puede mencionarse la propuesta de innovación docente Clara Peeters y sus otras historias para el tratamiento didáctico, entre otros, del papel de las mujeres en la Historia y su inclusión curricular en este mismo nivel educativo (Triviño-Cabrera, 2018).

Desde esta perspectiva, resulta fundamental cuestionar las estructuras existentes a fin de proponer procesos de enseñanza donde la experiencia histórica de las mujeres y la diversidad de género impulsen la promoción de habilidades de pensamiento social, crítico y creativo y, por lo tanto, el empoderamiento del alumnado en la construcción diversa de su identidad personal y social (Crocco, 2010, 2018; Vavrus, 2009; Barton, 2002). En este sentido, Colley (2019) considera que es necesario que el profesorado sea capaz de abordar las representaciones del alumnado sobre el género y el feminismo, fomentar la identificación y descripción de los sistemas de poder, analizar su impacto en las identidades de género, y comprender los mecanismos de la opresión institucionalizada en las narrativas históricas del alumnado, con el objeto de trabajar y alcanzar la igualdad de género en los procesos de enseñanza y aprendizaje en el ámbito específico de los estudios sociales. Con este propósito, resulta necesario el diseño de instrumentos válidos, como el aportado en la presente investigación, específicamente dirigidos a analizar, de forma operativa e integral, las narrativas históricas generadas por los y las propias estudiantes, a partir de su experiencia temporal y actuación social del presente.

\section{Agradecimientos}

Esta investigación ha sido realizada al amparo del proyecto Education and Democratic Hope. Rethinking Social Studies Education in changing times (PID2019-107383RB-I00), financiado por el Ministerio de Ciencia e Innovación, en el marco del Programa Estatal de Generación de Conocimiento y Fortalecimiento Científico y Tecnológico del sistema de I+D+i orientada a los Retos de la Sociedad. Este proyecto se encuentra vinculado con el Grupo de Investigación Reconocido en Didáctica de la Historia y de las Ciencias Sociales (DHISO) de la Universidad de Burgos.

\section{Conflicto de intereses}

El autor y la autora declaran no tener ningún conflicto de intereses. Los financiadores no tuvieron ningún papel en el diseño del estudio, en la recopilación, análisis o interpretación de datos, en la redacción del manuscrito o en la decisión de publicar los resultados.

\section{Contribuciones de los autores}

Conceptualización, D.O.-S. y D.H.-S.; metodología, D.O.-S.; software, D.O.-S.; validación, D.0.S. y D.H.-S.; análisis formal, D.O.-S.; investigación, D.O.-S. y D.H.-S.; recursos, D.O.-S. y D.H.-S.; conservación de datos, D.O.-S. y D.H.-S.; redacción - preparación del borrador original, D.O.-S.; redacción - revisión y revisión y edición, D.O.-S. y D.H.-S.; visualización, D.O.-S. y D.H.-S.; supervisión, D.O.-S. y D.H.-S.; administración del proyecto, D.O.-S. y D.H.-S.

El autor y la autora declaran que han leído y aceptado la versión publicada del manuscrito. 


\section{Referencias}

Andréu, J. (2000). Las técnicas de Análisis de Contenido: Una revisión actualizada. Fundación Centro Estudios Andaluces - Universidad de Granada, 10(2), 1-34.

Arange, D. (2019). Formar para enseñar Ciencias Sociales en la escuela primaria desde un enfoque didáctico con perspectiva de género. En M.J. Hortas y A. Dias (Eds.), Enseñar y aprender Didáctica de las Ciencias Sociales: la formación del profesorado desde una perspectiva sociocrítica (pp.243-252). Escola Superior de Educação / Instituto Politécnico de Lisboa - Asociación Universitaria de Profesorado de Didáctica de las Ciencias Sociales.

Asián, R., Cabeza, F., \& Rodríguez Sosa, V. (2015). Formación en Género en la Universidad: ¿Materia de asignaturas específicas o de educación transversal? Revista Historia de la Educación Latinoamericana, 17(24), 35-54. https://doi.org/10.19053/01227238.3310

Azorín, C. M. (2015). Análisis de la política de género en España: hacia una educación inclusiva y coeducadora. En A.B. Mirete, \& R. Nortes (Ed.), Investigación e Innovación: una constante necesaria en la formación del profesorado (pp. 1-12). Universidad de Murcia.

Balteiro, I., \& Roig-Marín, A. (2015). Reflexiones sobre la integración de cuestiones de género en la enseñanza universitaria. En J.D. Álvarez Teruel, M.T. Tortosa, \& N. Pellín (Ed.), Investigación y propuestas innovadoras de Redes UA para la mejora docente (pp. 851-870). Universidad de Alicante - Instituto de Ciencias de la Educación (ICE).

Barton, K. (2002). Masculinity and Schooling. Theory and Research in Social Education, 30(2), 306-312. https://doi.org/10.1080/00933104.2002.10473198

Campos, M.A. (2005). Construcción del conocimiento. Plaza y Valdés.

Cartes, D., Chávez, C., \& Menesses, B. (2019). Evidencias de la conciencia histórica en las planificaciones de profesores de Secundaria de Chile. En M.J. Hortas, A. Dias, \& N. de Alba (Eds.), Enseñar y aprender Didáctica de las Ciencias Sociales: la formación del profesorado desde una perspectiva sociocrítica (pp. 70-80). Escola Superior de Educação / Instituto Politécnico de Lisboa - Asociación Universitaria de Profesorado de Didáctica de las Ciencias Sociales.

Cea D’Ancona, M.A. (1996). Metodología cuantitativa. Estrategias y técnicas de investigación social. Síntesis.

Cerri, L.F. (2013). A formação de professores de História no Brasil: antecedentes e panorama atual. Revista história, histórias, 1(2), 167-186.

Colley, L. (2019). (Un)Restricting feminism: High school students' definitions of gender and feminism in the context of the historic struggle for women's rights. Theory \& Research in Social Education, 47(3), 426-455. https://doi.org/10.1080/00933104.2019.1593268

Conde J., \& Armas, X. (2019). Representaciones sociales del alumnado de Educación Primaria. Estudio por medio de la asociación de palabras. Revista Interuniversitaria de formación de Formación del Profesorado, 94(33.2), 187201.

Creswell, J.W. (2009). Research design: Qualitative, quantitative, and mixed 
approaches. SAGE.

Crocco, M.S. (2010). Using Literature to Teach about Others, the case of Shabanu. In

W. Parker (Ed.), Social Studies Today: Research and Practice (pp. 175-182).

Routledge.

Crocco, M.S. (2018). Gender and sexuality in history education. In S.A. Metzger, \& L. McArthur (Eds.), The Wiley International Handbook of History Teaching and Learning (pp. 335-364). Wiley. https://doi.org/10.1002/9781119100812.ch13

Denzin, N.K., \& Lincoln, Y.S. (Eds.) (2018). The SAGE Handbook of Qualitative Research. SAGE.

Esses, V.M., \& Maio, G.R. (2002). Expanding the assessment of attitude components and structure: The benefits of open-ended measures. European Review of Social Psychology, 12(1), 71-101.

Fernández Valencia, A. (2015). Género y enseñanza de la Historia. En M.A. Domínguez Arranz, \& R.M. Sáez (Eds.), Género y enseñanza de la Historia: silencios y ausencias en la construcción del pasado (pp. 29-55). Sílex.

González-Monfort, N., Pagès, J. \& Santisteban, A. (2015). ¿Quién protagoniza la Historia? análisis de los relatos históricos del alumnado de educación Primaria y Secundaria. En A.M. Hernández, C.R. García, \& J.L. de la Montaña, (Eds.), Una enseñanza de las Ciencias Sociales para el futuro: recursos para trabajar la invisibilidad de personas, lugares y temáticas (pp. 801-812). Universidad de Extremadura - Asociación Universitaria de Profesorado de Didáctica de las Ciencias Sociales.

Hernández, R., Fernández, C., \& Baptista, M.P. (2010). Metodología de la investigación. McGraw-Hill.

Krippendorff, K. (1990): Metodología del análisis de contenido: teoría y práctica. Paidós.

Kuckartz, U. (2014). Qualitative Text Analysis: A Guide to Methods, Practice and Using Software. SAGE.

Laville, C. (1999). A guerra das narrativas: debates e ilusões em torno do ensino de História. Revista brasileira da História, 19(38), 125-138.

Létourneau, J., \& Caritey, C. (2008). L'histoire du Québec racontée par les élèves de 4e et 5e secondaire: L'impact apparent du cours d'histoire nationale dans la structuration d'une mémoire historique collective chez les jeunes Québécois. Revue d'histoire de l'Amérique française, 62(1), 69-93.

Libarkin, J.C., \& Kurdziel, J.P. (2002). Research methodologies in science education: The Qualitative-Quantitative Debate. Journal of Geoscience Education, 50(1), 7886.

Mayring, Ph. (2010). Qualitative Inhaltsanalyse. Beltz Verlag.

McEwan, H., \& Egan, K. (Comp.). (1995). Narrative in teaching, learning and research. Teachers College Press - Columbia University.

McLaren, P., \& Kincheloe, J. (2015). Pedagogía crítica. De qué hablamos, dónde estamos. Graó.

Moliner, P., \& Abric, J.C. (2014). Central core theory. In G. Sammut, E. Andreouli, G. Gaskell, \& J. Valsiner (Eds.), The Cambridge Handbook of Social Representations (pp. 83-95). Cambridge University Press. 
Montoro Ríos, F. J., \& Muñoz Leiva, F. (2005). La medición de la fiabilidad interjuez en la codificación de preguntas abiertas: Una propuesta metodológica [Conference]. XV Jornadas Luso-Españolas de Gestión Científica, Sevilla.

Moreira, A.I., Duarte, P., \& Alves, L.A. (2019). Da consciência histórica à formação de professores (de Ciências Sociais). En M.J. Hortas, A. Dias, \& N. de Alba (Eds.), Enseñar y aprender Didáctica de las Ciencias Sociales: la formación del profesorado desde una perspectiva sociocrítica (pp.81-89). Escola Superior de Educação / Instituto Politécnico de Lisboa - Asociación Universitaria de Profesorado de Didáctica de las Ciencias Sociales.

Neuendorf, K.A. (2002). The Content Analysis Guidebook. SAGE.

Ortega-Sánchez, D. (2017). Las mujeres en la enseñanza de la Historia y de las Ciencias Sociales. Estudio de caso en formación inicial de maestros y maestras de Educación Primaria [Tesis Doctoral, Universitat Autònoma de Barcelona]. https://bit.ly/3btokXn

Ortega-Sánchez, D. (2019). Teaching Gender in the History Classroom: An Investigation into the Initial Training of Primary Education Teachers. Educ. Sci., 9(2), 114. https://doi.org/10.3390/educsci9020114

Ortega-Sánchez, D., \& Heras-Sevilla, D. (2020). Design and Initial Validation of the Scales Epistemology, Methodology and Gender in Taught History (EMG), and Women in History (WH) for the Evaluation of Gender Competence of Social Science Teachers in Training. Universal Journal of Educational Research, 8(5), 1831-1841. https://doi.org/10.13189/ujer.2020.080519

Ortega-Sánchez, D., \& Pagès, J. (2018). La construcción de identidades de género en la enseñanza de la Historia escolar: un estudio a partir de las narrativas históricas de los futuros y futuras docentes de Educación Primaria. En E. López, C.R. García, \& M. Sánchez (Eds.), Buscando nuevas formas de enseñar: Investigar para innovar en Didáctica de las Ciencias Sociales (pp. 89-100). Universidad de Valladolid - Asociación Universitaria de Profesorado de Didáctica de las Ciencias Sociales

Ortega-Sánchez, D., Marolla, J., \& Heras-Sevilla, D. (2020). Invisibilidades sociales, identidades de género y competencia narrativa en los discursos históricos del alumnado de Educación Primaria. En E.J. Díez Gutiérrez, \& J.R. Rodríguez Fernández (Eds.), Educación para el Bien Común: hacia una práctica crítica, inclusiva y comprometida socialmente (pp. 89-103). Octaedro

Pagès J., \& Sant, E. (2012). Las mujeres en la enseñanza de la Historia: ¿hasta cuándo serán invisibles? Cad. Pesq. Cdhis, Uberlândia, 25(1), 91-117. https://doi.org/10.14393/cdhis.v25i1.20957

Pesavento, S.J. (2000). Fronteiras da ficção. Diálogos da História com a literatura. Revista de História das Ideias, 21, 33-57.

Regina, S. (2019). Formar professores em tempo de obscurantismos: novis e venhos temas e problemas do campo da Didática das Ciências Sociais. En M.J. Hortas, A. Dias, \& N. de Alba (Eds.), Enseñar y aprender Didáctica de las Ciencias Sociales: la formación del profesorado desde una perspectiva sociocrítica (pp. 18-39). Escola Superior de Educação / Instituto Politécnico de Lisboa - Asociación Universitaria de Profesorado de Didáctica de las Ciencias Sociales. 
Sáiz, J., \& López, R. (2016). Narrativas nacionales históricas de estudiantes y profesorado en formación. Revista de Educación, 374, 118-141. https://doi.org/10.4438/1988-592X-RE-2016-374-328

Sant, E., González-Monfort, N., Pagès, J., Santisteban, A., \& Oller, M. (2014a). 'La historia de Cataluña está protagonizada por Franco y los Segadores'. Los protagonistas de la historia de Cataluña en las narraciones de los futuros docentes catalanes. En J. Pagès y A. Santisteban (Eds.). Una mirada al pasado y un proyecto de futuro: investigación e innovación en didáctica de las ciencias sociales (pp. 305-313). Universitat Autònoma de Barcelona- Asociación Universitaria de Profesorado de Didáctica de las Ciencias Sociales.

Sant, E., Pagès, J., Santisteban, A., González-Monfort, N., \& Oller, M. (2014b). ¿Cómo podemos analizar la competencia narrativa del alumnado en el aprendizaje de la Historia? Clío \& Asociados, 18-19, 166-182.

Seixas, P., \& Morton, T. (2013). The big six historical thinking concepts. Nelson.

Shahnaz, A., Fatima, S., \& Qadir, S. (2020). 'The myth that children can be anything they want': gender construction in Pakistani children literature. Journal of Gender Studies, 29(4),

470-482. https://doi.org/10.1080/09589236.2020.1736529

Stanley, W. (2010). Social Studies and the Social Order, Transmission or Transformation? In W. Parker (Ed.), Social Studies Today: Research and Practice (pp. 17-24). Routledge.

Triviño-Cabrera, L. (2018). "Clara Peeters y sus otras historias»: propuesta de innovación docente para afrontar resistencias y prejuicios en la formación del profesorado del máster de profesorado de educación secundaria y bachillerato. Revista Complutense de Educación, 29(3). https://doi.org/10.5209/rced.53645

Triviño-Cabrera, L., Bernárdez-Rodal, A., \& Velázquez-Felipe, A. (2020). The @Filosoclips project: teaching feminist philosophy through popular culture in Spain. Gender and Education, 1-15. https://doi.org/10.1080/09540253.2020.1837348

Vavrus, M. (2009). Sexuality, schooling, and teacher identity formation: A critical pedagogy for teacher education. Teaching and Teacher Education, 25(3), 383390. https://doi.org/10.1016/j.tate.2008.09.002 